\title{
The obstacle problem for a class of hypoelliptic ultraparabolic equations
}

\author{
Marco Di Francesco, Andrea Pascucci and Sergio Polidoro \\ Dipartimento di Matematica, Università di Bologna *
}

\begin{abstract}
We prove that the obstacle problem for a non-uniformly parabolic operator of Kolmogorov type, with Cauchy (or Cauchy-Dirichlet) boundary conditions, has a unique strong solution $u$. We also show that $u$ is a solution in the viscosity sense.
\end{abstract}

\section{Introduction}

We consider a class of second order differential operators of Kolmogorov type

$$
L u(z):=\sum_{i, j=1}^{m} a_{i j}(z) \partial_{x_{i} x_{j}} u(z)+\sum_{i=1}^{m} b_{i}(z) \partial_{x_{i}} u(z)+\sum_{i, j=1}^{N} b_{i j} x_{i} \partial_{x_{j}} u(z)-\partial_{t} u
$$

where $z=(x, t) \in \mathbb{R}^{N+1}, 1 \leq m \leq N$ and $b_{i j} \in \mathbb{R}$ for every $i, j=1, \ldots, N$. We assume:

H1 the coefficients $a_{i j}=a_{j i}$ and $b_{i}$ are bounded continuous functions for $i, j=1, \ldots, m$.

Moreover, there exists a positive constant $\Lambda$ such that

$$
\Lambda^{-1}|\zeta|^{2} \leq \sum_{i, j=1}^{m} a_{i j}(z) \zeta_{i} \zeta_{j} \leq \Lambda|\zeta|^{2}, \quad \zeta \in \mathbb{R}^{m}, z \in \mathbb{R}^{N+1}
$$

H2 the operator

$$
K u:=\sum_{i=1}^{m} \partial_{x_{i} x_{i}} u+\sum_{i, j=1}^{N} b_{i j} x_{i} \partial_{x_{j}} u-\partial_{t} u
$$

is hypoelliptic, i.e. every distributional solution of $K u=f$ is a smooth solution, whenever $f$ is smooth.

*Piazza di Porta S. Donato 5, 40126 Bologna (Italy), polidoro@dm.unibo.it, pascucci@dm.unibo.it, difrance@dm.unibo.it 
Hypothesis $\mathbf{H 2}$ is equivalent to the classical Hörmander condition [19]:

$$
\operatorname{rank} \text { Lie }\left(\partial_{x_{1}}, \ldots, \partial_{x_{m}}, Y\right)(z)=N+1, \quad \forall z \in \mathbb{R}^{N+1}
$$

where Lie $\left(\partial_{x_{1}}, \ldots, \partial_{x_{m}}, Y\right)$ denotes the Lie algebra generated by the vector fields $\partial_{x_{1}}, \ldots, \partial_{x_{m}}$ and

$$
Y:=\sum_{i, j=1}^{N} b_{i j} x_{i} \partial_{x_{j}}-\partial_{t}
$$

We explicitly remark that uniformly parabolic operators satisfy $\mathbf{H} \mathbf{1}$ and $\mathbf{H} \mathbf{2}$ with $m=N$. We also recall (cf. [25]) that $\mathbf{H 2}$ is equivalent to the existence of a basis of $\mathbb{R}^{N}$ with respect to which the matrix $B=\left(b_{i j}\right)$ assumes the following block form

$$
\left(\begin{array}{ccccc}
* & B_{1} & 0 & \ldots & 0 \\
* & * & B_{2} & \ldots & 0 \\
\vdots & \vdots & \vdots & \ddots & \vdots \\
* & * & * & \ldots & B_{r} \\
* & * & * & \ldots & *
\end{array}\right)
$$

where the blocks $*$ are constant and arbitrary, $B_{j}$ is a $m_{j-1} \times m_{j}$ matrix of rank $m_{j}$ and

$$
m=: m_{0} \geq m_{1} \geq \ldots \geq m_{r} \geq 1, \quad m_{0}+m_{1}+\ldots+m_{r}=N .
$$

This paper is mainly concerned with the obstacle problem

$$
\begin{cases}\max \{L u+a u-f, \varphi-u\}=0, & \text { in } \left.\mathbb{R}^{N} \times\right] 0, T[ \\ u(\cdot, 0)=g, & \text { in } \mathbb{R}^{N}\end{cases}
$$

where $a, f, g$ are bounded continuous functions. The assumptions on the obstacle function $\varphi$ will be specified in $\mathbf{H 4}$ in Section 3: we require that $\varphi$ is locally Lipschitz continuous and satisfies a weak convexity condition with respect to the variables $x_{1}, \ldots, x_{m}$.

Apart from the obvious importance in PDEs' theory, obstacle problems have natural theoretical interest in stochastic control. Moreover they appear in several applications in physics, biology and mathematical finance. Specifically, one of the best-known problems in finance is that of determining the arbitrage-free price of American style options. Precisely, we consider a financial model where the dynamic of the state variables is described by a $N$-dimensional diffusion process $X=\left(X_{t}^{x}\right)$ which is a solution to the stochastic differential equation

$$
d X_{t}^{x}=B X_{t}^{x} d t+\sigma\left(t, X_{t}^{x}\right) d W_{t}, \quad X_{t_{0}}^{t_{0}, x}=x,
$$

where $\left(x, t_{0}\right) \in \mathbb{R}^{N} \times[0, T]$ and $W$ denotes a $m$-dimensional Brownian motion, $m \leq N$. An American option with payoff $\varphi$ is a contract granting the holder to receive the payment of the sum $\varphi\left(X_{t}\right)$ at a time $t \in[0, T]$, which is chosen by the holder. Then, according 
to theory of modern finance (cf., for instance, Peskir and Shiryaev [32]) the arbitragefree price, at time 0 , of the American option is given by the following optimal stopping problem

$$
u(x, t)=\sup _{t \leq \tau \leq T} E\left[\varphi\left(X_{\tau}^{t, x}\right)\right],
$$

where the supremum is taken over all stopping times $\tau \in[t, T]$ of $X$. The main result in [31] is that the function $u$ in (1.6) is a solution of a problem in the form (1.4) where the obstacle function $\varphi$ corresponds to the payoff of the option and $L$ is the Kolmogorov operator associated to the diffusion $X$ :

$$
L=\frac{1}{2} \sum_{i, j=1}^{m}\left(\sigma \sigma^{T}\right)_{i j} \partial_{x_{i} x_{j}}+\langle B x, \nabla\rangle-\partial_{t} .
$$

In the uniformly parabolic case $m=N$, the valuation of American options has been studied starting from the papers by Bensoussan [4] and Karatzas [22] using a probabilistic approach based on Snell envelopes and by Jaillet, Lamberton e Lapeyre [20] using variational techniques. However there are significant classes of American options, commonly traded in financial markets, whose corresponding diffusion process $X$ is associated with Kolmogorov type operators that are not uniformly parabolic. Two remarkable examples are provided by Asian style options (cf., for instance, Barucci, Polidoro and Vespri [3]) and by some recent stochastic volatility model with dependence on the past (cf. Hobson and Rogers [18], Di Francesco and Pascucci [9], Foschi and Pascucci [12]). A general theory for these financial instruments is not available. Actually, the several papers on American Asian options available in literature (cf., for instance, Rogers and Shi [34], Barraquand and Pudet [2], Barles [1], Hansen and Jorgensen [17], Meyer [29], Jiang and Dai [21], Marcozzi [28], Dai e Kwok [8]) mainly consider numerical issues.

The aim of this paper, and of the related work [31], is to develop a rigorous theory for the obstacle problem (1.4) and the optimal stopping problem (1.6). The main results of this paper are the existence of a strong solution to the obstacle problem in a bounded cylindrical domain (cf. Theorem 3.1) and in the strip $\left.\mathbb{R}^{N} \times\right] 0, T[$ (cf. Theorem 4.1). We recall that, even in the standard framework of uniformly parabolic operators, problem (1.4) generally does not admit a solution in the classical sense. Three main approaches are used to tackle the existence problem: these are based on the notion of variational solution (cf. Bensoussan and Lions [5], Kinderlehrer and Stampacchia [23]), strong solution (cf. Friedman [13], [15]) and, more recently, viscosity solution (cf. Crandall, Ishii and Lions [7], Barles [1]). Since operator (1.1) appears in non-divergence form, we adapt a classical penalization technique to find a unique strong solution to (1.4), obtained as the limit of solutions to a suitable class of non-linear problems.

We also show in Theorem 5.2 that the strong solutions are viscosity solutions and in [31] it is proved that the function $u$ defined in (1.6) is a strong solution to the obstacle problem (1.4). As a consequence, the solutions to American option problem provided by means of different methodologies in [1], [21] and [8], must coincide. 
Concerning the regularity, we emphasize that any strong solution $u$ is Hölder continuous with its first order derivatives $\partial_{x_{1}} u, \ldots, \partial_{x_{m}} u$ (see Section 2). This result, combined with the above remark, improves the regularity of viscosity solutions and solutions of the optimal stopping problem (1.6), obtained by probabilistic techniques. Starting from these results, we aim to investigate the regularity properties of the obstacle problem in a forthcoming study.

This paper is organized as follows. In Section 2 we set the notations and introduce the functional setting suitable for the study of the regularity properties of the operator (1.1): specifically, our study is cast in the framework of analysis on Lie groups. The proofs of some of the results stated in this section are postponed to Section 6. In Sections 3 and 4 we prove the existence and uniqueness of the solution to the obstacle problem in bounded domains and in $\mathbb{R}^{N}$, respectively. The main result of Section 5 states that the strong solutions are also viscosity solutions.

\section{Functional analysis on Lie groups}

Since the works by Folland [11], Rothschild and Stein [35], Nagel, Stein and Wainger [30], it is known that the natural framework for the study of operators satisfying the Hörmander condition is the analysis on Lie groups. The Lie group structure related to Kolmogorov operators has been first studied by Lanconelli and Polidoro in [25]. The explicit expression of the group law is defined by

$$
(\xi, \tau) \circ(x, t)=(x+E(t) \xi, t+\tau),
$$

where $E(t)=e^{-t B^{T}}$ and $B^{T}$ denotes the transpose of $B$. The solutions of the equation $K u=0$, with $K$ as in (1.2), have the remarkable property of being invariant with respect to the left translations defined by (2.1):

$$
K u(\zeta \circ z)=(K u)(\zeta \circ z)
$$

or equivalently, if $K u=f$, then

$$
\sum_{i=1}^{m} \partial_{x_{i} x_{i}} u(\zeta \circ z)+\sum_{i, j=1}^{N} b_{i j} x_{i} \partial_{x_{j}} u(\zeta \circ z)-\partial_{t} u(\zeta \circ z)=f(\zeta \circ z)
$$

for every $z=(x, t), \zeta=(\xi, \tau) \in \mathbb{R}^{N+1}$. Moreover, if and only if the $*$-blocks in (1.3) are null, operator $K$ is homogeneous of degree two with respect to the dilations defined by

$$
D(\lambda)=\operatorname{diag}\left(\lambda I_{m_{0}}, \lambda^{3} I_{m_{1}}, \ldots, \lambda^{2 r+1} I_{m_{r}}, \lambda^{2}\right)
$$

where $I_{m_{j}}$ denotes the $m_{j} \times m_{j}$ identity matrix. The number

$$
Q+2=m_{0}+3 m_{1}+\ldots+(2 r+1) m_{r}+2
$$


is usually called $D(\lambda)$-homogeneous dimension of $\mathbb{R}^{N+1}$. Then $\mathcal{G}_{B}=\left(\mathbb{R}^{N+1}, \circ,(D(\lambda))\right)$ is a homogeneous Lie group only determined by $B$.

We also recall the definition of a $D(\lambda)$-homogeneous norm: for every $z \in \mathbb{R}^{N+1} \backslash\{0\}$, we define $\|z\|=\rho$ if $\rho$ is the unique positive solution of

$$
\frac{x_{1}^{2}}{\rho^{2 q_{1}}}+\frac{x_{2}^{2}}{\rho^{2 q_{2}}}+\ldots+\frac{x_{n}^{2}}{\rho^{2 q_{N}}}+\frac{t^{2}}{\rho^{4}}=1
$$

and $q_{1}, \ldots q_{N}$ are the integers such that

$$
D(\lambda)(x, t)=\left(\lambda^{q_{1}} x_{1}, \lambda^{q_{2}} x_{2}, \ldots, \lambda^{q_{N}} x_{N}, \lambda^{2} t\right) .
$$

Some functional spaces related to the Lie group $\mathcal{G}_{B}$ are defined as follows. Let $\Omega$ be a domain of $\mathbb{R}^{N+1}$ and $p \geq 1$. We set

$$
\mathcal{S}^{p}(\Omega)=\left\{u \in L^{p}(\Omega): \partial_{x_{i}} u, \partial_{x_{i} x_{j}} u, Y u \in L^{p}(\Omega), i, j=1, \ldots, m\right\}
$$

and

$$
\|u\|_{\mathcal{S}^{p}(\Omega)}=\|u\|_{L^{p}(\Omega)}+\sum_{i=1}^{m}\left\|\partial_{x_{i}} u\right\|_{L^{p}(\Omega)}+\sum_{i, j=1}^{m}\left\|\partial_{x_{i} x_{j}} u\right\|_{L^{p}(\Omega)}+\|Y u\|_{L^{p}(\Omega)}
$$

We say that $u \in \mathcal{S}_{\text {loc }}^{p}(\Omega)$ if $u \in \mathcal{S}^{p}(O)$ for every compact $O \subset \Omega$. Moreover, we denote respectively by $C_{B}^{\alpha}(\Omega), C_{B}^{1, \alpha}(\Omega)$ and $C_{B}^{2, \alpha}(\Omega)$ the Hölder spaces defined by the following norms:

$$
\begin{aligned}
\|u\|_{C_{B}^{\alpha}(\Omega)} & =\sup _{\Omega}|u|+\sup _{\substack{z, \zeta, \in \Omega \\
z \neq \zeta}} \frac{|u(z)-u(\zeta)|}{\left\|\zeta^{-1} \circ z\right\|^{\alpha}}, \\
\|u\|_{C_{B}^{1, \alpha}(\Omega)} & =\|u\|_{C_{B}^{\alpha}(\Omega)}+\sum_{i=1}^{m}\left\|\partial_{x_{i}} u\right\|_{C_{B}^{\alpha}(\Omega)}, \\
\|u\|_{C_{B}^{2, \alpha}(\Omega)} & =\|u\|_{C_{B}^{1, \alpha}(\Omega)}+\sum_{i, j=1}^{m}\left\|\partial_{x_{i} x_{j}} u\right\|_{C_{B}^{\alpha}(\Omega)}+\|Y u\|_{C_{B}^{\alpha}(\Omega)} .
\end{aligned}
$$

Note that any $u \in C_{B}^{\alpha}(\Omega)$ is Hölder continuous in the usual sense since

$$
\left\|\zeta^{-1} \circ z\right\| \leq c|z-\zeta|^{\frac{1}{2 r+1}}
$$

Next we state some results extending the usual embedding theorems and a priori interior estimates. In the case of homogeneous Kolmogorov operators they have been proved in several papers (cf. Bramanti, Cerutti and Manfredini [6], Manfredini and Polidoro [27], Manfredini [26]). In the Appendix we generalize these results to the non-homogeneous case. Hereafter when we claim that a constant depends on $L$ we mean that it depends on $N, m, B$ and the constant $\Lambda$ in $\mathbf{H 1}$. 
Theorem 2.1 (Embedding theorem). Let $O, \Omega$ be bounded domains of $\mathbb{R}^{N+1}$ such that $O \subset \subset \Omega$ and $p>Q+2$. There exists a positive constant $c$, only dependent on $L, \Omega, O$ and $p$, such that

$$
\|u\|_{C_{B}^{1, \alpha}(O)} \leq c\|u\|_{\mathcal{S}^{p}(\Omega)}, \quad \alpha=1-\frac{Q+2}{p}
$$

for any $u \in \mathcal{S}^{p}(\Omega)$.

Theorem 2.2 (A priori $\mathcal{S}^{p}$ interior estimates). Let $O, \Omega$ be bounded domains of $\mathbb{R}^{N+1}$ such that $O \subset \subset \Omega$. There exists a positive constant $c$, only depending on $L, O, \Omega$ and $p$, such that

$$
\|u\|_{\mathcal{S}^{p}(O)} \leq c\left(\|u\|_{L^{p}(\Omega)}+\|L u\|_{L^{p}(\Omega)}\right)
$$

for every $u \in \mathcal{S}^{p}(\Omega), 1<p<\infty$.

In the sequel, we also use the following Schauder type estimate, proved by Di Francesco and Polidoro in [10]:

Theorem 2.3 (Interior Schauder estimate). Let $O, \Omega$ be bounded domains of $\mathbb{R}^{N+1}$, such that $O \subset \subset \Omega$. Let the coefficients $a_{i j}, b_{i} \in C_{B}^{\alpha}(\Omega)$. For any $u \in C_{B}^{2, \alpha}(\Omega)$ we have

$$
\|u\|_{C_{B}^{2, a}(O)} \leq c\left(\sup _{\Omega}|u|+\|L u\|_{C_{B}^{\alpha}(\Omega)}\right)
$$

for some positive constant $c$ only depending on $\alpha, \Omega, O, L,\left\|a_{i j}\right\|_{C_{B}^{a}(\Omega)}$ and $\left\|b_{i}\right\|_{C_{B}^{a}(\Omega)}$.

\section{Obstacle problem on bounded domains}

In this section, we prove the existence and uniqueness of a strong solution to the obstacle problem

$$
\left\{\begin{array}{l}
\max \{L u+a u-f, \varphi-u\}=0, \quad \text { in } H(T):=H \times] 0, T[, \\
\left.u\right|_{\partial_{P} H(T)}=g,
\end{array}\right.
$$

where $H$ is a bounded domain in $\mathbb{R}^{N}$ and

$$
\partial_{P} H(T):=\partial H(T) \backslash(H \times\{T\})
$$

denotes the parabolic boundary of $H(T)$. We say that $u \in \mathcal{S}_{\mathrm{loc}}^{1}(H(T)) \cap C(\overline{H(T)})$ is a strong solution to problem (3.1) if the differential inequality is satisfied a.e. in $H(T)$ and the boundary datum is attained pointwisely.

We assume that $H(T)$ is regular in the sense that at every point of its parabolic boundary there exists a barrier function. Precisely,

H3 for any $\zeta \in \partial_{P} H(T)$ there exists a neighborhood $V$ of $\zeta$ and a $C^{2}$ function

$$
w: V \cap \overline{H(T)} \longrightarrow \mathbb{R},
$$

such that 
i) $L w \leq-1$ in $V \cap H(T)$;

ii) $w(z)>0$ in $V \cap \overline{H(T)} \backslash\{\zeta\}$ and $w(\zeta)=0$.

Next we state the assumption on the obstacle function $\varphi$.

$\mathbf{H} 4 \varphi$ is a Lipschitz continuous function on $\overline{H(T)}$ and there exists a constant $C \in \mathbb{R}$ such that

$$
\sum_{i, j=1}^{m} \zeta_{i} \zeta_{j} \partial_{x_{i} x_{j}} \varphi \geq C|\zeta|^{2}, \quad \text { in } H(T), \zeta \in \mathbb{R}^{m},
$$

in the distributional sense, that is

$$
\sum_{i, j=1}^{m} \zeta_{i} \zeta_{j} \int_{H(T)} \partial_{x_{i} x_{j}} \psi(z) \varphi(z) d z \geq C|\zeta|^{2} \int_{H(T)} \psi(z) d z
$$

for any $\zeta \in \mathbb{R}^{m}$ and $\psi \in C_{0}^{\infty}(H(T)), \psi \geq 0$.

We explicitly note that $C^{2}$ functions satisfy assumption $\mathbf{H} 4$ as well as Lipschitz continuous function that are convex with respect to the first $m$ variables.

The main result of this section is the following

Theorem 3.1. Assume H1, H2, H3 and H4. Let $g \in C\left(\partial_{P} H(T)\right)$, such that $g \geq \varphi$, and $f, a \in C \cap L^{\infty}(H(T))$. Then there exists a strong solution $u$ of the problem (3.1). Moreover, for every $p \geq 1$ and $O$ compact subset of $H(T)$, there exists a positive constant $c$, only depending on $L, O, H(T), p$ and on the $L^{\infty}$-norms of $f, g, \varphi$ and $a$, such that

$$
\|u\|_{\mathcal{S}^{p}(O)} \leq c
$$

We prove Theorem 3.1 by adapting a standard penalization technique (cf., for instance, Friedman [14]). Therefore we consider a family $\left(\beta_{\varepsilon}\right)_{\varepsilon \in] 0,1[}$ of smooth functions: fixed $\varepsilon>0$, $\beta_{\varepsilon}$ is an increasing function, bounded with its first order derivatives, such that

$$
\beta_{\varepsilon}(0)=0, \quad \beta_{\varepsilon}(s) \leq \varepsilon, \quad s>0,
$$

and

$$
\lim _{\varepsilon \rightarrow 0} \beta_{\varepsilon}(s)=-\infty, \quad s<0 .
$$

For $\delta \in] 0,1$, we denote by $L^{\delta}$ the operator obtained from $L$ by mollifying the coefficients $a_{i j}$ and $b_{i}$. We also denote by $\varphi^{\delta}$ (respectively, $\left.a^{\delta}, f^{\delta}\right)$ the regularization ${ }^{1}$ of $\varphi($ resp. $a, f)$. Since $g \geq \varphi$ in $\partial_{P} H(T)$ we have

$$
g^{\delta}:=g+\lambda \delta \geq \varphi^{\delta}, \quad \text { in } \partial_{P} H(T),
$$

\footnotetext{
${ }^{1}$ We may suitably extend $\varphi, a, f$ by continuity in a neighborhood of $H(T)$.
} 
where $\lambda$ is the Lipschitz constant of $\varphi$. Then we consider the penalized problem

$$
\left\{\begin{array}{l}
L^{\delta} u+a^{\delta} u=f^{\delta}+\beta_{\varepsilon}\left(u-\varphi^{\delta}\right), \quad \text { in } H(T), \\
\left.u\right|_{\partial_{P} H(T)}=g^{\delta} .
\end{array}\right.
$$

As a first step, we prove that a classical solution of (3.4) exits.

Theorem 3.2. Assume H1, H2 and H3. Let $g \in C\left(\partial_{P} H(T)\right)$ and let $h=h(z, u)$ be a Lipschitz continuous function on $\overline{H(T)} \times \mathbb{R}$. Then there exists a classical solution $u \in C_{B}^{2, \alpha}(H(T)) \cap C(\overline{H(T)})$ of problem

$$
\left\{\begin{array}{l}
L^{\delta} u=h(\cdot, u), \quad \text { in } H(T), \\
\left.u\right|_{\partial_{P} H(T)}=g .
\end{array}\right.
$$

Moreover, there exists a positive constant $c$, only depending on $h$ and $H(T)$, such that

$$
\sup _{H(T)}|u| \leq e^{c T}\left(1+\|g\|_{L^{\infty}}\right) .
$$

Proof. We use a monotone iterative method. We set

$$
u_{0}(x, t)=e^{c t}\left(1+\|g\|_{L^{\infty}}\right)-1,
$$

where $c$ is a positive constant such that $|h(z, u)| \leq c(1+|u|)$ for $(z, u) \in \overline{H(T)} \times \mathbb{R}$. Then we define recursively the sequence $\left(u_{j}\right)_{j \in \mathbb{N}}$ by

$$
\left\{\begin{array}{l}
L^{\delta} u_{j}-\lambda u_{j}=h\left(\cdot, u_{j-1}\right)-\lambda u_{j-1}, \quad \text { in } H(T), \\
\left.u_{j}\right|_{\partial_{P} H(T)}=g
\end{array}\right.
$$

where $\lambda$ is the Lipschitz constant of $h$. Let us recall that the linear problem (3.6) has a unique classical solution $\left.\left.C_{B}^{2, \alpha}(H(T)) \cap C(\overline{H(T)}), \alpha \in\right] 0,1\right]$, by Theorem 4.1 in [10].

Next we prove by induction that $\left(u_{j}\right)$ is a decreasing sequence. By the maximum principle we have $u_{1} \leq u_{0}$ : indeed

$$
L^{\delta}\left(u_{1}-u_{0}\right)-\lambda\left(u_{1}-u_{0}\right)=h\left(\cdot, u_{0}\right)-L^{\delta} u_{0}=h\left(\cdot, u_{0}\right)+c\left(1+u_{0}\right) \geq 0,
$$

and $u_{1} \leq u_{0}$ on $\partial_{P} H(T)$. Now, fixed $j \in \mathbb{N}$, we assume the inductive hypothesis $u_{j} \leq u_{j-1}$; then, recalling that $\lambda$ is the Lipschitz constant of $h$, we have

$$
L^{\delta}\left(u_{j+1}-u_{j}\right)-\lambda\left(u_{j+1}-u_{j}\right)=h\left(\cdot, u_{j}\right)-h\left(\cdot, u_{j-1}\right)-\lambda\left(u_{j}-u_{j-1}\right) \geq 0 .
$$

Moreover $u_{j+1}=u_{j}$ on $\partial_{P} H(T)$, so that the maximum principle implies $u_{j+1} \leq u_{j}$. The same argument shows that $u_{j}$ is bounded from below by $-u_{0}$. In conclusion, for $j \in \mathbb{N}$, we have

$$
-u_{0} \leq u_{j+1} \leq u_{j} \leq u_{0}
$$


Let us denote by $u$ the pointwise limit of $\left(u_{j}\right)$ in $\overline{H(T)}$. Since $u_{j}$ is a solution of (3.6) and by the uniform estimate (3.7), we can apply Theorem 2.1 and 2.2 to conclude that, for any compact subset $O$ of $H(T)$ and $\alpha \in] 0,1\left[,\left\|u_{j}\right\|_{C_{B}^{1, \alpha}(O)}\right.$ is bounded by a constant only dependent on $L, H(T), O, \alpha$ and $\lambda$. Hence by the Schauder interior estimate (2.5), we deduce that $\left\|u_{j}\right\|_{C_{B}^{2, \alpha}(O)}$ is bounded uniformly in $j \in \mathbb{N}$. It follows that $\left(u_{j}\right)_{j \in \mathbb{N}}$ admits a subsequence (denoted by itself) that locally converges in $C_{B}^{2, \alpha}$. Thus passing at limit in (3.6) as $j \rightarrow \infty$, we have

$$
L^{\delta} u=h(\cdot, u), \quad \text { in } H(T),
$$

and $\left.u\right|_{\partial_{p} H(T)}=g$.

In order to prove that $u \in C(\overline{H(T)})$, we use the standard argument of barrier functions. Fixed $\zeta \in \partial_{P} H(T)$ and $\varepsilon>0$, let $V$ be an open neighborhood of $\zeta$ such that

$$
|g(z)-g(\zeta)| \leq \varepsilon, \quad z \in V \cap \partial_{P} H(T)
$$

and a barrier function $w$ as in $\mathbf{H 3}$ is defined. We set

$$
v^{ \pm}(z)=g(\zeta) \pm\left(\varepsilon+k_{\varepsilon} w(z)\right)
$$

where $k_{\varepsilon}$ is a suitably large positive constant, independent of $j$, such that

$$
L^{\delta}\left(u_{j}-v^{+}\right) \geq h\left(\cdot, u_{j-1}\right)-\lambda\left(u_{j-1}-u_{j}\right)+k_{\varepsilon} \geq 0,
$$

and $u_{j} \leq v^{+}$on $\partial(V \cap H(T))$. The maximum principle yields $u_{j} \leq v^{+}$on $V \cap H(T)$; analogously we have $u_{j} \geq v^{-}$on $V \cap H(T)$, and letting $j \rightarrow \infty$ we get

$$
g(\zeta)-\varepsilon-k_{\varepsilon} w(z) \leq u(z) \leq g(\zeta)+\varepsilon+k_{\varepsilon} w(z), \quad z \in V \cap H(T) .
$$

Then

$$
g(\zeta)-\varepsilon \leq \liminf _{z \rightarrow \zeta} u(z) \leq \limsup _{z \rightarrow \zeta} u(z) \leq g(\zeta)+\varepsilon, \quad z \in V \cap H(T),
$$

which proves the thesis since $\varepsilon$ is arbitrary. Eventually the bound (3.5) is a direct consequence of the maximum principle and (3.7).

Proof of Theorem 3.1. By Theorem 3.2 with

$$
h(\cdot, u)=f^{\delta}+\beta_{\varepsilon}\left(u-\varphi^{\delta}\right)-a^{\delta} u,
$$

the penalized problem (3.4) has a classical solution $u_{\varepsilon, \delta} \in C_{B}^{2, \alpha}(H(T)) \cap C(\overline{H(T)})$. In the sequel we assume $a \leq 0$ : up to a standard transformation, this is not restrictive. We first show that

$$
\left|\beta_{\varepsilon}\left(u_{\varepsilon, \delta}-\varphi^{\delta}\right)\right| \leq \widetilde{c}
$$

for a constant $\widetilde{c}$ independent of $\varepsilon$ and $\delta$. Since $\beta_{\varepsilon} \leq \varepsilon$ we only have to prove the estimate from below. Let us denote by $\zeta$ a minimum point of the function $\beta_{\varepsilon}\left(u_{\varepsilon, \delta}-\varphi^{\delta}\right) \in C(\overline{H(T)})$ 
and assume $\beta_{\varepsilon}\left(u_{\varepsilon, \delta}(\zeta)-\varphi^{\delta}(\zeta)\right) \leq 0$, since otherwise there is nothing to prove. If $\zeta \in$ $\partial_{P} H(T)$ then

$$
\beta_{\varepsilon}\left(g^{\delta}(\zeta)-\varphi^{\delta}(\zeta)\right) \geq \beta_{\varepsilon}(0)=0 .
$$

If $\zeta \in H(T)$, since $\beta_{\varepsilon}$ is an increasing function, $u_{\varepsilon, \delta}-\varphi^{\delta}$ also assumes the (negative) minimum at $\zeta$ : then

$$
L^{\delta} u_{\varepsilon, \delta}(\zeta)-L^{\delta} \varphi^{\delta}(\zeta) \geq 0 \geq-a(\zeta)\left(u_{\varepsilon, \delta}(\zeta)-\varphi^{\delta}(\zeta)\right) .
$$

Now, by $\mathbf{H} 4, L^{\delta} \varphi^{\delta}(\zeta)$ is bounded from below by a constant independent of $\delta$. Therefore, by (3.9), we have

$$
\begin{aligned}
\beta_{\varepsilon}\left(u_{\varepsilon, \delta}(\zeta)-\varphi^{\delta}(\zeta)\right) & =L^{\delta} u_{\varepsilon, \delta}(\zeta)+a^{\delta}(\zeta) u_{\varepsilon, \delta}(\zeta)-f^{\delta}(\zeta) \\
& \geq L^{\delta} \varphi^{\delta}(\zeta)+a^{\delta}(\zeta) \varphi^{\delta}(\zeta)-f^{\delta}(\zeta) \geq \widetilde{c},
\end{aligned}
$$

with $\widetilde{c}$ independent of $\varepsilon, \delta$. This concludes the proof of (3.8).

By the maximum principle, we have

$$
\left\|u_{\varepsilon, \delta}\right\|_{\infty} \leq\|g\|_{L^{\infty}}+T\left(\|f\|_{L^{\infty}}+\widetilde{c}\right) .
$$

Therefore, using the $\mathcal{S}^{p}$ interior estimates and (3.8), (3.10) we infer that, for every $O \subset \subset$ $H(T)$ and $p \geq 1$, the norm $\left\|u_{\varepsilon, \delta}\right\|_{\mathcal{S}^{p}(O)}$ is bounded uniformly in $\varepsilon$ and $\delta$. It follows that the $\left(u_{\varepsilon, \delta}\right)$ converges as $\varepsilon, \delta \rightarrow 0$, weakly in $\mathcal{S}^{p}$ (and in $C_{B}^{1, \alpha}$ ) on compact subsets of $H(T)$ to a function $u$. Moreover

$$
\limsup _{\varepsilon, \delta \rightarrow 0} \beta_{\varepsilon}\left(u_{\varepsilon, \delta}-\varphi^{\delta}\right) \leq 0
$$

so that $L u+a u \leq f$ a.e. in $H(T)$. On the other hand, $L u+a u=f$ a.e. in the set $\{u>\varphi\}$.

Finally, repeating the argument based on barrier functions at the end of the proof of Theorem 3.2, we conclude that $u \in C(\overline{H(T)})$ and $u=g$ on $\partial_{P} H(T)$.

We close this section by proving a comparison result.

Proposition 3.3. Let $u$ be a strong solution of (3.1) and $v \in \mathcal{S}_{\text {loc }}^{1}(H(T)) \cap C(\overline{H(T)})$ be such that

$$
\left\{\begin{array}{l}
\max \{L v+a v-f, \varphi-v\} \leq 0, \quad \text { a.e. in } H(T), \\
\left.v\right|_{\partial_{P} H(T)} \geq g .
\end{array}\right.
$$

Then $u \leq v$ in $H(T)$. In particular the solution to (3.1) is unique.

Proof. By contradiction, suppose that the open set

$$
D:=\{z \in H(T) \mid u(z)>v(z)\}
$$

is nonempty. Then, since $u>v \geq \varphi$ in $D$, we have

$$
L u+a u=f, \quad L v+a u \leq f \quad \text { in } D,
$$

and $u=v$ on $\partial D$. Then the maximum principle implies $u \geq v$ in $D$ and we get a contradiction. 


\section{Obstacle problem on unbounded domains}

In this section, we prove the existence of a unique strong solution to the obstacle problem

$$
\begin{cases}\max \{L u+a u-f, \varphi-u\}=0, & \text { in } \left.S_{T}:=\mathbb{R}^{N} \times\right] 0, T[, \\ u(\cdot, 0)=g, & \text { in } \mathbb{R}^{N} .\end{cases}
$$

We say that $\bar{u}$ is a (strong) super-solution of problem (4.1) if $\bar{u} \in \mathcal{S}_{\text {loc }}^{1}\left(S_{T}\right) \cap C\left(\mathbb{R}^{N} \times[0, T[)\right.$ and

$$
\begin{cases}\max \{L \bar{u}+a \bar{u}-f, \varphi-\bar{u}\} \leq 0, & \text { a.e. in } S_{T}, \\ \bar{u}(\cdot, 0) \geq g, & \text { in } \mathbb{R}^{N} .\end{cases}
$$

and that $\underline{u}$ is a (strong) sub-solution if the conditions (4.2) hold with the inequalities reversed. Finally $u$ is a strong solution of problem (4.1), if $u$ is super and sub-solution. We assume

H5 $\varphi$ is a locally Lipschitz continuous function on $\overline{S_{T}}$ such that, for every convex and compact subset $M$ of $S_{T}$, the convexity condition (3.2) holds with real constant $C$ dependent on $M$.

Our main result is the following

Theorem 4.1. Assume H1, H2, H5 and let $a, f \in C\left(S_{T}\right)$ with $a \leq a_{0}$ for some $a_{0} \in \mathbb{R}$ and $g \in C\left(\mathbb{R}^{N}\right)$ such that $g \geq \varphi(\cdot, 0)$. If there exists a strong super-solution $\bar{u}$ of problem (4.1) then there also exists a strong solution $u$ of (4.1) such that $u \leq \bar{u}$ in $S_{T}$.

The existence of a supersolution is ensured for instance if $g, \varphi$ are bounded functions and $f \geq 0$. In this case we can simply set $\bar{u}(x, t):=e^{a_{0} t} \max \left\{\|g\|_{\infty},\|\varphi\|_{\infty}\right\}$.

Proof. We prove the theorem by solving a sequence of obstacle problems on regular bounded domains. Specifically we define the cylindrical domain $H_{n}(T), n \in \mathbb{N}$, as follows: let $e_{1}=(1,0, \ldots, 0)$ be the first vector of the canonical basis of $\mathbb{R}^{N}$ and denote by $B_{n}\left(x_{0}\right)$ the Euclidean ball of $\mathbb{R}^{N}$ with center at $x_{0} \in \mathbb{R}^{N}$ and radius $n$. We define

$$
O_{n}=B_{n+1}\left(e_{1}\right) \cap B_{n+1}\left(-e_{1}\right),
$$

and, for every $T>0$,

$$
\left.H_{n}(T)=O_{n} \times\right] 0, T[.
$$

For such a domain a barrier function is defined at every point of the parabolic boundary

$$
\partial_{P} H_{n}(T)=\left(O_{n} \times\{0\}\right) \cup\left(\partial O_{n} \times[0, T]\right) .
$$

See, for instance [24] or [10] for details. Note that the sequence $\left(H_{n}(T)\right)_{n \in \mathbb{N}}$ covers $S_{T}$.

For every $n \in \mathbb{N}$, we consider a cut-off function $\chi_{n} \in C\left(\mathbb{R}^{N} ;[0,1]\right)$ such that $\chi_{n}(x)=1$ if $x \in O_{n-\frac{1}{2}}$ and $\chi_{n}(x)=0$ if $x \notin O_{n}$, and set

$$
g_{n}(x, t)=\chi_{n}(x) g(x)+\left(1-\chi_{n}(x)\right) \bar{u}(x, t), \quad(x, t) \in S_{T} .
$$


By Theorem 3.1, for every $n \in \mathbb{N}$, there exists a strong solution $u_{n}$ of

$$
\left\{\begin{array}{l}
\max \{L u+a u-f, \varphi-u\}=0, \quad \text { in } H_{n}(T), \\
\left.u\right|_{\partial_{P} H_{n}(T)}=g_{n}
\end{array}\right.
$$

By Proposition 3.3 it is straightforward to prove that

$$
\varphi \leq u_{n+1} \leq u_{n} \leq \bar{u}, \quad \text { in } H_{n}(T)
$$

In order to conclude, it is sufficient to use the same arguments as in the proofs of Theorems 3.1 and 4.1 , based on the a priori $\mathcal{S}_{\text {loc }}^{p}$ interior estimates and the barrier functions.

\section{$5 \quad$ Viscosity solutions}

In this section we prove that any strong solution to (4.1) solves the same problem in the viscosity sense as well. This is almost standard to verify by using the well-known fact that viscosity solutions pass to the limit under uniform convergence. Adopting the notations of the User's Guide [7], we set

$$
F(z, u, p, X)=p_{N+1}-\operatorname{trace}(A(z) X)-\sum_{i, j=1}^{N} b_{i j} x_{i} p_{j}-a(z) u+f(z), \quad z=(x, t)
$$

for $p \in \mathbb{R}^{N+1}$ (which stands for the gradient in $\mathbb{R}^{N+1}$ w.r.t. the variables $(x, t)$ ), $X$ symmetric $(N+1) \times(N+1)$ matrix and

$$
A_{i j}= \begin{cases}a_{i j} & i, j=1, \ldots, m \\ 0 & \text { otherwise }\end{cases}
$$

We denote by $u_{\delta}$ the classical solution of the regularized and penalized equation

$$
L^{\delta} u_{\delta}=f^{\delta}+\beta_{\delta}\left(u_{\delta}-\varphi^{\delta}\right), \quad \text { in } S_{T},
$$

subject to the initial condition $u(\cdot, 0)=g$ in $\mathbb{R}^{N}$. Moreover let $F^{\delta}$ be the operator formally defined as $F$ with $A, a, f$ respectively replaced by $A^{\delta}, a^{\delta}, f^{\delta}$.

Then $u_{\delta}$ is a viscosity solution of (5.1) i.e. $u_{\delta}$ is continuous and it is a sub- and super-solution of the equation in the sense that

$$
F^{\delta}\left(z, u_{\delta}(z), p, X\right)+\beta_{\delta}\left(u_{\delta}(z)-\varphi_{\delta}(z)\right) \leq 0, \quad z \in S_{T},(p, X) \in J^{2,+} u_{\delta}(z),
$$

and

$$
F^{\delta}\left(z, u_{\delta}(z), p, X\right)+\beta_{\delta}\left(u_{\delta}(z)-\varphi_{\delta}(z)\right) \geq 0, \quad z \in S_{T},(p, X) \in J^{2,-} u_{\delta}(z) .
$$


In (5.2)-(5.3) $J^{2,+} u(z)$ and $J^{2,-} u(z)$ denote respectively the second order super- and subjet of $u$ at $z$ defined by

$$
\begin{aligned}
& \qquad J^{2,+} u(z)=\left\{\left(D \psi(z), D^{2} \psi(z)\right) \mid \psi \in C^{2}\left(\mathbb{R}^{N+1}\right) \text { and } u-\psi \text { has a local maximum at } z\right\}, \\
& \text { and } J^{2,-} u(z)=-J^{2,+}(-u)(z) \text {. }
\end{aligned}
$$

For what follows we need the following result which is contained [7], Proposition 4.3.

Lemma 5.1. Let $u \in C\left(S_{T}\right), z \in S_{T},\left(p^{+}, X^{+}\right) \in J^{2,+} u(z)$ and $\left(p^{-}, X^{-}\right) \in J^{2,-} u(z)$. Suppose that $\left(u_{\delta}\right)$ is a family of continuous functions, uniformly convergent as $\delta \rightarrow 0$ to $u$ in a neighborhood of $z$. Then there exist sequences $\left(\delta_{n}\right)$ in $\mathbb{R},\left(z_{n}\right)$ in $S_{T},\left(p_{n}^{+}, X_{n}^{+}\right) \in$ $J^{2,+} u_{\delta_{n}}\left(z_{n}\right)$ and $\left(p_{n}^{-}, X_{n}^{-}\right) \in J^{2,-} u_{\delta_{n}}\left(z_{n}\right)$ such that

$$
\lim _{n \rightarrow \infty}\left(z_{n}, p_{n}^{+}, X_{n}^{+}, p_{n}^{-}, X_{n}^{-}\right)=\left(z, p^{+}, X^{+}, p^{-}, X^{-}\right) .
$$

Theorem 5.2. Any strong solution of (4.1) is also a viscosity solution.

Proof. Since $u \in C\left(\bar{S}_{T}\right)$ and $u \geq \varphi$, it suffices to show that

i) $L u \leq f$ on $S_{T}$ in the viscosity sense, that is

$$
F(z, u(z), p, X) \geq 0, \quad \text { for all } \quad z \in S_{T},(p, X) \in J^{2,-} u(z)
$$

ii) $L u=f$ in the viscosity sense on $\{u>\varphi\}$.

To this end we consider a sequence $\left(u_{\delta_{n}}\right)$ of solutions to the regularized and penalized problem, locally uniformly convergent to $u$. Fixed $z \in S_{T}$ and $\left(p^{-}, X^{-}\right) \in J^{2,-} u(z)$, we consider a sequence $\left(z_{n}, p_{n}^{-}, X_{n}^{-}\right) \in S_{T} \times J^{2,-} u_{\delta_{n}}\left(z_{n}\right)$ as in Lemma 5.1. Then we have

$$
\lim _{n \rightarrow \infty} F_{n}\left(z_{n}, u_{\delta_{n}}\left(z_{n}\right), p_{n}^{-}, X_{n}^{-}\right)=F\left(z, u(z), p^{-}, X^{-}\right)
$$

and, by (5.1),

$$
\lim _{n \rightarrow \infty} F_{n}\left(z_{n}, u_{\delta_{n}}\left(z_{n}\right), p_{n}^{-}, X_{n}^{-}\right)=-\lim _{n \rightarrow \infty} \beta_{n}\left(u_{\delta_{n}}\left(z_{n}\right)-\varphi_{n}\left(z_{n}\right)\right) \geq 0,
$$

and this proves (5.4).

Analogously, fixed $z \in S_{T}$ such that $u(z)>\varphi(z)$ and $\left(p^{+}, X^{+}\right) \in J^{2,+} u(z)$, by Lemma 5.1 we may select a sequence $\left(z_{n}, p_{n}^{+}, X_{n}^{+}\right) \in S_{T} \times J^{2,+} u_{\delta_{n}}\left(z_{n}\right)$ such that $u_{\delta_{n}}\left(z_{n}\right)>\varphi_{n}\left(z_{n}\right)$ and then we conclude

$$
F\left(z, u(z), p^{+}, X^{+}\right)=\lim _{n \rightarrow \infty} F_{n}\left(z_{n}, u_{\delta_{n}}\left(z_{n}\right), p_{n}^{+}, X_{n}^{+}\right)=-\lim _{n \rightarrow \infty} \beta_{n}\left(u_{\delta_{n}}\left(z_{n}\right)-\varphi_{n}\left(z_{n}\right)\right)=0
$$




\section{Proof of Theorems 2.1 and 2.2}

The proof of Theorem 2.2 relies on some representation formulas in terms of a parametrix, i.e. of the fundamental solution of a suitable homogeneous operator $L_{z}$. We first recall some known fact about the fundamental solution of the homogeneous operator, and about the related singular integrals.

We next fix some notations useful in the sequel. We denote by $B_{0}$ the matrix obtained by replacing every block " $*$ " in (1.3) with a block matrix of zeros:

$$
B_{0}=\left(\begin{array}{ccccc}
0 & B_{1} & 0 & \ldots & 0 \\
0 & 0 & B_{2} & \ldots & 0 \\
\vdots & \vdots & \vdots & \ddots & \vdots \\
0 & 0 & 0 & \ldots & B_{r} \\
0 & 0 & 0 & \ldots & 0
\end{array}\right)
$$

The Lie group related to $B_{0}$ will be denoted as

$$
z \cdot w=\left(y+E_{0}(s) x, t+s\right), \quad \text { where } \quad E_{0}(s)=e^{s B_{0}},
$$

for every $z=(x, t), w=(y, s) \in \mathbb{R}^{N} \times \mathbb{R}$. We define the sets $\mathcal{I}_{0}, \mathcal{I}_{1}, \mathcal{I}$ by letting

$$
\mathcal{I}_{0}=\left\{(i, j): q_{i}=q_{j}\right\}, \quad \mathcal{I}_{1}=\left\{(i, j): q_{i}<q_{j}\right\}, \quad \mathcal{I}=\mathcal{I}_{0} \cup \mathcal{I}_{1},
$$

where $q_{1}, \ldots, q_{N}$ are the integers introduced in (2.2). Note that the coefficients $b_{i j}$ of the set $\mathcal{I}_{0}$ are the ones corresponding to the "*" blocks of the principal diagonal of the matrix $B$ and that the coefficients in $\mathcal{I}_{1}$ are the ones in the blocks below the principal diagonal. We point out that

$$
\langle y, B D v(w)\rangle=\left\langle y, B_{0} D v(w)\right\rangle+\sum_{(i, j) \in \mathcal{I}} b_{i j} y_{i} \partial_{y_{j}} v(w)
$$

We finally denote by $L_{z}$, the homogeneous operator with the coefficients $a_{i j}$ frozen at $z \in \mathbb{R}^{N+1}$ :

$$
L_{z} v(w):=\sum_{i, j=1}^{m} a_{i j}(z) \partial_{y_{i} y_{j}}^{2} v(w)+\left\langle y, B_{0} D v(w)\right\rangle-\partial_{s} v(w) .
$$

and by $\Gamma_{z}$ its fundamental solution

$$
\Gamma_{z}(\xi, \tau ; y, s)=\frac{(4 \pi)^{-\frac{N}{2}}}{\sqrt{\operatorname{det} \mathcal{C}_{z}(\tau-s)}} e^{-\frac{1}{4}\left\langle\mathcal{C}_{z}^{-1}(\tau-s)\left(\xi-E_{0}(\tau-s) y\right), \xi-E_{0}(\tau-s) y\right\rangle}
$$

for $\tau>s$ and $\Gamma_{z}(\xi, \tau, y, s)=0$ if $\tau \leq s$, where $\mathcal{C}_{z}(t)$ is the $N \times N$ matrix

$$
\mathcal{C}_{z}(t)=\int_{0}^{t} E_{0}(s)\left(\begin{array}{ll}
A_{z} & 0 \\
0 & 0
\end{array}\right) E_{0}^{T}(s) d s, \quad A_{z}=\left(a_{i j}(z)\right)_{i, j=1, \ldots, m} .
$$


It is known that $\Gamma_{z}$ is homogeneous of degree $-Q$ with respect to the dilations $D(\lambda)_{\lambda>0}$, i.e.

$$
\Gamma_{z}(D(\lambda) \zeta, 0)=\lambda^{-Q} \Gamma_{z}(\zeta, 0), \quad \forall \zeta \in \mathbb{R}^{N+1} \backslash\{0\}, \lambda>0 .
$$

Moreover the derivative $\partial_{\xi_{j}} \Gamma_{z}(\zeta, 0)$ is homogeneous of degree $-Q-q_{j},(j=1, \ldots, N)$ and $\partial_{\xi_{j} \xi_{k}}^{2} \Gamma_{z}(\zeta, 0)$ is homogeneous of degree $-Q-q_{j}-q_{k},(j, k=1, \ldots, N)$.

In the sequel we rely on some known results about potential estimates and singular integral defined in terms of $\Gamma_{z}$ and its derivatives (see [11]). Let $G_{\alpha}$ denote a homogeneous function of degree $\alpha-Q-2$, with $\alpha \in\left[0, Q+2\left[\right.\right.$, and let $f \in L^{p}\left(\mathbb{R}^{N+1}\right)$ for some $\left.p \in\right] 1,+\infty[$. If $\alpha \neq 0$, then the potential

$$
G_{\alpha}(f)(w):=\int_{\mathbb{R}^{N+1}} G_{\alpha}\left(\zeta^{-1} \cdot w\right) f(\zeta) d \zeta
$$

defines a function belonging to $L^{q}\left(\mathbb{R}^{N+1}\right)$, with $\frac{1}{q}=\frac{1}{p}-\frac{\alpha}{Q+2}$, and there exists a positive constant $c_{\alpha}$, depending on $G_{\alpha}$ and $p$, such that

$$
\left\|G_{\alpha}(f)\right\|_{L^{q}\left(\mathbb{R}^{N+1}\right)} \leq c_{\alpha}\|f\|_{L^{p}\left(\mathbb{R}^{N+1}\right)} .
$$

If $\alpha=0$, we also require that $G_{0}$ has the vanishing property

$$
\int_{\left\|\zeta^{-1}\right\|=1} G_{0}\left(\zeta^{-1}\right) d \sigma(\zeta)=0
$$

In that case the Principal Value of the singular integral

$$
P . V . \int_{\mathbb{R}^{N+1}} G_{0}\left(\zeta^{-1} \cdot w\right) f(\zeta) d \zeta:=\lim _{\varepsilon \rightarrow 0} \int_{\left\|\zeta^{-1}\right\| \geq \varepsilon} G_{0}\left(\zeta^{-1} \cdot w\right) f(\zeta) d \zeta
$$

is a function in $L^{p}\left(\mathbb{R}^{N+1}\right)$, and there exists a positive constant $c_{0}=c_{0}\left(G_{0}, p\right)$ such that

$$
\left\|P . V . \int_{\mathbb{R}^{N+1}} G_{0}\left(\zeta^{-1} \cdot w\right) f(\zeta) d \zeta\right\|_{L^{p}\left(\mathbb{R}^{N+1}\right)} \leq c_{0}\|f\|_{L^{p}\left(\mathbb{R}^{N+1}\right)} .
$$

In (6.6) and (6.7) $\zeta^{-1}$ denotes the inverse of $\zeta$ with respect to the operation ".".

In the sequel, we will use a representation formula in terms of the homogeneous functions $\partial_{y_{i} y_{j}}^{2} \Gamma_{z}(y, s, \xi, \tau)(i, j=1, \ldots, m)$ computed at $(y, s)=(0,0)$. By the invariance with respect to the translation "." we have $\Gamma_{z}(w, \zeta)=\Gamma_{z}\left(\zeta^{-1} \cdot w, 0\right)=: \Gamma_{z}\left(\zeta^{-1} \cdot w\right)$ then, for the sake of brevity, in the sequel we will use the following notation:

$$
\partial_{y_{i} y_{j}}^{2} \Gamma_{z}\left(\zeta^{-1}\right):=\left.\partial_{y_{i} y_{j}}^{2} \Gamma_{z}\left(\zeta^{-1} \cdot(y, s)\right)\right|_{(y, s)=(0,0)} .
$$

It is known that $\partial_{y_{i} y_{j}}^{2} \Gamma_{z}\left(\zeta^{-1}\right)$ is a homogeneous function of degree $-Q-2$ and has the vanishing property (see [6]). We next show that the same result holds for $\partial_{y_{i} y_{j} \xi_{h}}^{3}\left(\xi_{k} \Gamma_{z}\left(\zeta^{-1}\right)\right)$, for every index $(h, k) \in \mathcal{I}_{0}$. It is easy to see that $\partial_{y_{i} y_{j} \xi_{h}}^{3}\left(\xi_{k} \Gamma_{z}\left(\zeta^{-1}\right)\right)$ is a homogeneous 
function of degree $-Q-2$, for any $(h, k) \in \mathcal{I}_{0}$. To prove the vanishing property, we use the same argument used in the proof of Theorem 2.2 in [6]: we use the "polar" change of variables (formula (1.7) in [6]) and the fact that $\partial_{y_{i} y_{j} \xi_{h}}^{3}\left(\xi_{k} \Gamma_{z}\left(\zeta^{-1}\right)\right)$ is homogeneous of degree $-Q-2$ and we get

$$
\int_{a \leq\left\|\zeta^{-1}\right\| \leq b} \partial_{y_{i} y_{j} \xi_{h}}^{3}\left(\xi_{k} \Gamma_{z}\left(\zeta^{-1}\right)\right) d \zeta=\log \left(\frac{b}{a}\right) \int_{\left\|\zeta^{-1}\right\|=1} \partial_{y_{i} y_{j} \xi_{h}}^{3}\left(\xi_{k} \Gamma_{z}\left(\zeta^{-1}\right)\right) d \sigma(\zeta) .
$$

Hence, it is enough to prove that the first integral is zero. The divergence theorem gives

$$
\begin{aligned}
\int_{a \leq\left\|\zeta^{-1}\right\| \leq b} \partial_{y_{i} y_{j} \xi_{h}}^{3}\left(\xi_{k} \Gamma_{z}\left(\zeta^{-1}\right)\right) d \zeta= & \\
& \int_{\left\|\zeta^{-1}\right\|=b} \partial_{y_{i} y_{j}}^{2}\left(\xi_{k} \Gamma_{z}\left(\zeta^{-1}\right)\right) \nu_{h} d \sigma(\zeta)-\int_{\left\|\zeta^{-1}\right\|=a} \partial_{y_{i} y_{j}}^{2}\left(\xi_{k} \Gamma_{z}\left(\zeta^{-1}\right)\right) \nu_{h} d \sigma(\zeta),
\end{aligned}
$$

where $\nu_{h}$ is the $h$-th component of the outer normal to the surface $\left\{z \in \mathbb{R}^{N+1}:\left\|z^{-1}\right\|=\varepsilon\right\}$. Proceeding as in the proof of Lemma 2.10 in [10] and using the fact that $\partial_{y_{i} y_{j}}^{2}\left(\xi_{k} \Gamma_{z}\left(\zeta^{-1}\right)\right) \nu_{h}$ is homogeneous of degree $-Q$, we see that

$$
\int_{\left\|\zeta^{-1}\right\|=\varepsilon} \partial_{y_{i} y_{j}}^{2}\left(\xi_{k} \Gamma_{z}\left(\zeta^{-1}\right)\right) \nu_{h} d \sigma(\zeta)
$$

does not depend on $\varepsilon>0$. Hence, the first integral in (6.8) equals zero, and this proves the vanishing property of $\partial_{y_{i} y_{j} \xi_{h}}^{3}\left(\xi_{k} \Gamma_{z}\left(\zeta^{-1}\right)\right)$.

Lemma 6.1. Let $u \in C_{0}^{\infty}\left(\mathbb{R}^{N+1}\right)$. Then the following representation formula holds:

$$
\begin{aligned}
u_{x_{i} x_{j}}(z) & =-P . V \cdot \int_{\mathbb{R}^{N+1}} \partial_{y_{i} y_{j}}^{2} \Gamma_{z}\left(\zeta^{-1}\right)\left(\sum_{h, k=1}^{m}\left(a_{h k}(z)-a_{h k}(z \circ \zeta)\right) \partial_{\xi_{h} \xi_{k}}^{2} u(z \circ \zeta)+\right. \\
& \left.L u(z \circ \zeta)-a(z \circ \zeta) u(z \circ \zeta)-\sum_{h=1}^{m} b_{h}(z \circ \zeta) \partial_{\xi_{h}} u(z \circ \zeta)\right) d \zeta- \\
& \left(L u(z)-a(z) u(z)-\sum_{h=1}^{m} b_{h}(z) \partial_{\xi_{h}} u(z)\right) \int_{\left\|\zeta^{-1}\right\|=1} \partial_{y_{i}} \Gamma_{z}\left(\zeta^{-1}\right) \nu_{j} d \sigma(\zeta)+ \\
& \sum_{(h, k) \in \mathcal{I}_{0}} b_{h k} P . V . \int_{\mathbb{R}^{N+1}} \partial_{y_{i} y_{j} \xi_{h}}^{3}\left(\xi_{k} \Gamma_{z}\left(\zeta^{-1}\right)\right) u(z \circ \zeta) d \zeta,+ \\
& u(z) \sum_{(h, k) \in \mathcal{I}_{0}} b_{h k} \int_{\left\|\zeta^{-1}\right\|=1} \partial_{y_{i} y_{j}}^{2}\left(\xi_{k} \Gamma_{z}\left(\zeta^{-1}\right)\right) \nu_{h} d \sigma(\zeta)+ \\
& \sum_{(h, k) \in \mathcal{I}_{1}} b_{h k} \int_{\mathbb{R}^{N+1}} \partial_{y_{i} y_{j} \xi_{h}}^{3}\left(\xi_{k} \Gamma_{z}\left(\zeta^{-1}\right)\right) u(z \circ \zeta) d \zeta
\end{aligned}
$$

for every $z \in \mathbb{R}^{N+1}$ and for $i, j=1, \ldots, m$. 
Proof. Fix $z \in \mathbb{R}^{N+1}$ and set $v(w):=u(z \circ w)$. By using the invariance of $Y$ with respect to the translation "o", we get $L_{z} v(w)=g(w)$, where (also using (6.3))

$$
\begin{aligned}
g(w)= & \sum_{h, k=1}^{m}\left[a_{h k}(z)-a_{h k}(z \circ w)\right] \partial_{y_{h} y_{k}}^{2} v(w)+\sum_{(h, k) \in \mathcal{I}} b_{h k} y_{k} \partial_{y_{h}} v(w)+ \\
& L u(z \circ w)-a(z \circ w) v(w)-\sum_{h=1}^{m} b_{h}(z \circ w) \partial_{y_{h}} v(w) .
\end{aligned}
$$

To prove (6.10), we rely on the usual representation formulas for $v$ and its derivatives

$$
\begin{gathered}
v(w)=-\int_{\mathbb{R}^{N+1}} \Gamma_{z}\left(\zeta^{-1} \cdot w\right) g(\zeta) d \zeta \\
v_{x_{i}}(w)=-\int_{\mathbb{R}^{N+1}} \partial_{y_{i}} \Gamma_{z}\left(\zeta^{-1} \cdot w\right) g(\zeta) d \zeta, \quad i=1, \ldots, m,
\end{gathered}
$$

where $\zeta^{-1}$ denotes the inverse of $\zeta$ with respect to the operation ".". We also consider the function

$$
V_{\epsilon}(w):=-\int_{\mathbb{R}^{N+1}} \eta_{\epsilon}\left(\zeta^{-1} \cdot w\right) \partial_{y_{i}} \Gamma_{z}\left(\zeta^{-1} \cdot w\right) g(\zeta) d \zeta
$$

It is clear that $V_{\epsilon}(w) \rightarrow v_{x_{i}}(w)$, as $\epsilon \rightarrow 0$. Besides, $V_{\epsilon} \in C^{\infty}$ and

$$
\partial_{y_{j}} V_{\epsilon}(w)=I_{1}(\varepsilon, z, w)+I_{2}(\varepsilon, z, w)+I_{3}(\varepsilon, z, w),
$$

where

$$
\begin{aligned}
I_{1}(\varepsilon, z, w) & :=-\sum_{h, k=1}^{m} \int_{\mathbb{R}^{N+1}} \partial_{y_{j}}\left(\eta_{\epsilon}\left(\zeta^{-1} \cdot w\right) \partial_{y_{i}} \Gamma_{z}\left(\zeta^{-1} \cdot w\right)\right)\left(a_{h k}(z)-a_{h k}(z \circ \zeta)\right) \\
I_{2}(\varepsilon, z, w) & :=-\sum_{\xi_{h} \xi_{k}}^{2} u(z \circ \zeta) d \zeta, \\
I_{3}(\varepsilon, z, w) \in \mathcal{I} & b_{h k} \int_{\mathbb{R}^{N+1}} \partial_{y_{j}}\left(\eta_{\epsilon}\left(\zeta^{-1} \cdot w\right) \partial_{y_{i}} \Gamma_{z}\left(\zeta^{-1} \cdot w\right)\right) \xi_{k} \partial_{\xi_{h}} u(z \circ \zeta) d \zeta \\
I_{\mathbb{R}^{N+1}} \partial_{y_{j}}\left(\eta_{\epsilon}\left(\zeta^{-1} \cdot w\right) \partial_{y_{i}} \Gamma_{z}\left(\zeta^{-1} \cdot w\right)\right)(L u(z \circ \zeta)-a(z \circ \zeta) u(z \circ \zeta)- & \left.\sum_{h=1}^{m} b_{h}(z \circ \zeta) \partial_{\xi_{h}} u(z \circ \zeta)\right) d \zeta .
\end{aligned}
$$


The same argument used in the proof of Theorem 2.4 of [6] gives

$$
\begin{aligned}
\lim _{\varepsilon \rightarrow 0} I_{1}(\varepsilon, z, 0)= & -\sum_{h, k=1}^{m} P . V \cdot \int_{\mathbb{R}^{N+1}} \partial_{y_{i} y_{j}}^{2} \Gamma_{z}\left(\zeta^{-1}\right)\left(a_{h k}(z)-a_{h k}(z \circ \zeta)\right) \partial_{\xi_{h} \xi_{k}}^{2} u(z \circ \zeta) d \zeta, \\
\lim _{\varepsilon \rightarrow 0} I_{3}(\varepsilon, z, 0)= & -P . V \cdot \int_{\mathbb{R}^{N+1}} \partial_{y_{i} y_{j}}^{2} \Gamma_{z}\left(\zeta^{-1}\right)(L u(z \circ \zeta)-a(z \circ \zeta) u(z \circ \zeta) \\
& \left.\sum_{h=1}^{m} b_{h}(z \circ \zeta) \partial_{\xi_{h}} u(z \circ \zeta)\right) d \zeta- \\
& \left(L u(z)-a(z) u(z)-\sum_{h=1}^{m} b_{h}(z) \partial_{\xi_{h}} u(z)\right) \int_{\left\|\zeta^{-1}\right\|=1} \partial_{y_{i}} \Gamma_{z}\left(\zeta^{-1}\right) \nu_{j} d \sigma(\zeta) .
\end{aligned}
$$

Moreover, the convergence is uniform with respect to $z$. We next consider the terms appearing in the sum in $I_{2}(\varepsilon, z, 0)$ :

$$
\begin{aligned}
\int_{\mathbb{R}^{N+1}} & \partial_{y_{j}}\left(\eta_{\epsilon}\left(\zeta^{-1}\right) \partial_{y_{i}} \Gamma_{z}\left(\zeta^{-1}\right)\right) \xi_{k} \partial_{\xi_{h}} u(z \circ \zeta) d \zeta= \\
& \int_{\left\|\zeta^{-1}\right\|<\varepsilon} \partial_{y_{j}}\left(\eta_{\epsilon}\left(\zeta^{-1}\right) \partial_{y_{i}} \Gamma_{z}\left(\zeta^{-1}\right)\right) \xi_{k} \partial_{\xi_{h}} u(z \circ \zeta) d \zeta+ \\
& \int_{\left\|\zeta^{-1}\right\| \geq \varepsilon} \partial_{y_{i} y_{j}}^{2} \Gamma_{z}\left(\zeta^{-1}\right) \xi_{k} \partial_{\xi_{h}} u(z \circ \zeta) d \zeta=: J_{1}(\varepsilon, z)+J_{2}(\varepsilon, z) .
\end{aligned}
$$

Concerning $J_{1}$, we note that the functions $\xi_{k} \partial_{y_{i}} \Gamma_{z}\left(\zeta^{-1}\right)$ and $\xi_{k} \partial_{y_{i} y_{j}}^{2} \Gamma_{z}\left(\zeta^{-1}\right)$ are homogeneous of degree $-Q-1+q_{k}$ and $-Q-2+q_{k}$, respectively, and that there exists a positive constant $c$ such that $\left|\partial_{y_{j}} \eta_{\epsilon}\left(\zeta^{-1}\right)\right| \leq \frac{c}{\varepsilon}$, for every $\zeta \in \mathbb{R}^{N+1}$. As a consequence we get

$$
J_{1}(\varepsilon, \cdot) \rightrightarrows 0, \quad \text { as } \quad \varepsilon \rightarrow 0 .
$$

By the divergence theorem we find

$$
\begin{aligned}
J_{2}(\varepsilon, z)=- & \int_{\left\|\zeta^{-1}\right\| \geq \varepsilon} \partial_{y_{i} y_{j} \xi_{h}}^{3}\left(\xi_{k} \Gamma_{z}\left(\zeta^{-1}\right)\right) u(z \circ \zeta) d \zeta+ \\
& \int_{\left\|\zeta^{-1}\right\|=\varepsilon} \xi_{k} \partial_{y_{i} y_{j}}^{2} \Gamma_{z}\left(\zeta^{-1}\right)(u(z \circ \zeta)-u(z)) \nu_{h} d \sigma(\zeta)+ \\
& u(z) \int_{\left\|\zeta^{-1}\right\|=\varepsilon} \xi_{k} \partial_{y_{i} y_{j}}^{2} \Gamma_{z}\left(\zeta^{-1}\right) \nu_{h} d \sigma(\zeta)=: J_{2}^{\prime}(\varepsilon, z)+J_{2}^{\prime \prime}(\varepsilon, z)+u(z) J_{2}^{\prime \prime \prime}(\varepsilon, z) .
\end{aligned}
$$

We first consider the indices $(h, k)$ in $\mathcal{I}_{0}$. Since the integral in (6.9) does not depend on $\varepsilon$, we have

$$
\left|J_{2}^{\prime \prime}(\varepsilon, z)\right| \leq \max _{\left\|\zeta^{-1}\right\|=\varepsilon}|u(z \circ \zeta)-u(z)| \int_{\left\|\zeta^{-1}\right\|=1}\left|\partial_{y_{i} y_{j} \xi_{h}}^{3}\left(\xi_{k} \Gamma_{z}\left(\zeta^{-1}\right)\right)\right| d \sigma(\zeta) .
$$


then, by also using the continuity of $v$, we get $J_{2}^{\prime \prime}(\varepsilon, z) \rightarrow 0$ as $\varepsilon \rightarrow 0$. Moreover, we have already observe that the function $\partial_{y_{i} y_{j} \xi_{h}}^{3}\left(\xi_{k} \Gamma_{z}\left(\zeta^{-1}\right)\right)$ is homogeneous of degree $-Q-2$ and has the vanishing property. Then, it is well defined the $P . V \cdot \int_{\mathbb{R}^{N+1}} \partial_{y_{i} y_{j} \xi_{h}}^{3}\left(\xi_{k} \Gamma_{z}\left(\zeta^{-1}\right)\right) u(z \circ$ $\zeta) d \zeta$

We next consider the indices $(h, k) \in \mathcal{I}_{1}$. In this case the functions $\partial_{y_{i} y_{j}}^{2}\left(\xi_{k} \Gamma_{z}\left(\zeta^{-1}\right)\right) \nu_{h}$ and $\partial_{y_{i} y_{j} \xi_{h}}^{3}\left(\xi_{k} \Gamma_{z}\left(\zeta^{-1}\right)\right)$ are homogeneous of degree $-Q+q_{k}-q_{h}$ and $-Q-2+q_{k}-q_{h}$, respectively. Since $q_{h}<q_{k}$, the function $\partial_{y_{i} y_{j} \xi_{h}}^{3}\left(\xi_{k} \Gamma_{z}\left(\zeta^{-1}\right)\right)$ is locally integrable, then

$$
\lim _{\varepsilon \rightarrow 0} J_{2}^{\prime}(\varepsilon, z)=\int_{\mathbb{R}^{N+1}} \partial_{y_{i} y_{j} \xi_{h}}^{3}\left(\xi_{k} \Gamma_{z}\left(\zeta^{-1}\right)\right) u(z \circ \zeta) d \zeta .
$$

On the other hand,

$$
\int_{\left\|\zeta^{-1}\right\|=\varepsilon} \xi_{k} \partial_{y_{i} y_{j}}^{2} \Gamma_{z}\left(\zeta^{-1}\right) \nu_{h} d \sigma(\zeta)=\varepsilon^{q_{k}-q_{h}} \int_{\left\|\zeta^{-1}\right\|=1} \xi_{k} \partial_{y_{i} y_{j}}^{2} \Gamma_{z}\left(\zeta^{-1}\right) \nu_{h} d \sigma(\zeta)
$$

then, $J_{2}^{\prime \prime}(\varepsilon, z) \rightarrow 0$ and $J_{2}^{\prime \prime \prime}(\varepsilon, z) \rightarrow 0$ as $\varepsilon \rightarrow 0$. We finally note that in each of the above limits the convergence is uniform with respect to $z$. Hence, also using (6.14), we find

$$
\begin{aligned}
\lim _{\varepsilon \rightarrow 0} I_{2}(\varepsilon, z, 0)= & \sum_{(h, k) \in \mathcal{I}_{0}} b_{h k} P . V \cdot \int_{\mathbb{R}^{N+1}} \partial_{y_{i} y_{j} \xi_{h}}^{3}\left(\xi_{k} \Gamma_{z}\left(\zeta^{-1}\right)\right) u(z \circ \zeta) d \zeta+ \\
& u(z) \sum_{(h, k) \in \mathcal{I}_{0}} b_{h k} \int_{\left\|\zeta^{-1}\right\|=1} \partial_{y_{i} y_{j}}^{2}\left(\xi_{k} \Gamma_{z}\left(\zeta^{-1}\right)\right) \nu_{h} d \sigma(\zeta)+ \\
& \sum_{(h, k) \in \mathcal{I}_{1}} b_{h k} \int_{\mathbb{R}^{N+1}} \partial_{y_{i} y_{j} \xi_{h}}^{3}\left(\xi_{k} \Gamma_{z}\left(\zeta^{-1}\right)\right) u(z \circ \zeta) d \zeta
\end{aligned}
$$

uniformly with respect to $z$. This identity accomplishes the proof of the Lemma.

Proof of Theorem 2.2. We first note that it is sufficient to prove the claim for any suitably small ball $B_{r}=B_{r}(z):=\left\{\zeta \in \mathbb{R}^{N+1} \mid\left\|\zeta^{-1} \circ z\right\|<r\right\}$, i.e.

$$
\|u\|_{\mathcal{S}^{p}\left(B_{\frac{r}{2}}\right)} \leq c\left(\|L u\|_{L^{p}\left(B_{r}\right)}+\|u\|_{L^{p}\left(B_{r}\right)}\right)
$$

Theorem 2.2 will follow by a standard covering argument.

In order to prove (6.15), we recall that the coefficients $a_{h k}$ are continuous, then, for every $\varepsilon>0$ and $z \in \mathbb{R}^{N+1}$ there exists $r>0$ such that

$$
\sup _{\|\zeta\| \leq r}\left|a_{h k}(z)-a_{h k}(z \circ \zeta)\right| \leq \varepsilon, \quad \forall h, k=1, \ldots m .
$$

If $v \in C^{\infty}\left(B_{r}(z)\right)$, then (6.7) yields

$$
\left\|P . V . \int_{\mathbb{R}^{N+1}} \partial_{y_{i} y_{j}}^{2} \Gamma_{z}\left(\zeta^{-1}\right)\left(a_{h k}(z)-a_{h k}(z \circ \zeta)\right) \partial_{\xi_{h} \xi_{k}}^{2} v(z \circ \zeta) d \zeta\right\|_{L^{p}\left(B_{r}\right)} \leq c_{0} \varepsilon\left\|\partial_{x_{h} x_{k}} v\right\|_{L^{p}\left(B_{r}\right)}
$$


for any $h, k=1, \ldots, m$. By using the above estimate, together with (6.6) and (6.7), in the representation formula (6.10), we find

$$
\left\|v_{x_{i} x_{j}}\right\|_{L^{p}\left(B_{r}\right)} \leq c_{1}\left(\varepsilon \sum_{h, k=1}^{m}\left\|v_{x_{h} x_{k}}\right\|_{L^{p}\left(B_{r}\right)}+\|L v\|_{L^{p}\left(B_{r}\right)}+\|v\|_{L^{p}\left(B_{r}\right)}+\sum_{h=1}^{m}\left\|v_{x_{h}}\right\|_{L^{p}\left(B_{r}\right)}\right),
$$

for some positive constant $c_{1}$. Then, if $\varepsilon$ is suitably small, we have

$$
\sum_{i, j=1}^{m}\left\|v_{x_{i} x_{j}}\right\|_{L^{p}\left(B_{r}\right)} \leq c_{2}\left(\|L v\|_{L^{p}\left(B_{r}\right)}+\|v\|_{L^{p}\left(B_{r}\right)}+\sum_{h=1}^{m}\left\|v_{x_{h}}\right\|_{L^{p}\left(B_{r}\right)}\right) .
$$

We finally note that $Y v=L v-\sum_{i, j=1}^{m} a_{i j} v_{x_{i} x_{j}}-\sum_{i=1}^{m} b_{i} v_{x_{i}}-a v$, and that the coefficients $a_{i j}, b_{i}$ and $a$ are bounded, so that we get

$$
\sum_{i, j=1}^{m}\left\|v_{x_{i} x_{j}}\right\|_{L^{p}\left(B_{r}\right)}+\|Y v\|_{L^{p}\left(B_{r}\right)} \leq c_{3}\left(\|L v\|_{L^{p}\left(B_{r}\right)}+\|v\|_{L^{p}\left(B_{r}\right)}+\sum_{h=1}^{m}\left\|v_{x_{h}}\right\|_{L^{p}\left(B_{r}\right)}\right),
$$

for every $v \in C^{\infty}\left(B_{r}(z)\right)$, with $r$ suitably small, where $c_{3}$ is a positive constant that may depend on $\varepsilon$.

We next prove (6.15). Consider a function $u \in \mathcal{S}^{p}\left(B_{r}\right)$. For any $\left.\rho \in\right] 0,1[$, we set $\rho^{\prime}=(1+\rho) / 2$ and we consider a function $\eta \in C^{\infty}\left(\mathbb{R}^{N+1}\right)$ such that $0 \leq \eta(\zeta) \leq 1$, $\eta(\zeta)=1$ if $\zeta \in B_{\rho r}, \eta(\zeta)=0$ if $\zeta \in B_{r} \backslash B_{\rho^{\prime} r}$. We may, and we do, assume that $\eta$ satisfies the following estimates

$$
\left|\partial_{x_{i}} \eta(\zeta)\right| \leq \frac{c_{4}}{\rho r}, \quad\left|\partial_{x_{i} x_{j}} \eta(\zeta)\right| \frac{c_{4}}{\rho^{2} r^{2}}, \quad|Y \eta(\zeta)| \leq \frac{c_{4}}{\rho^{2} r^{2}}, \quad \forall i, j=1, \ldots, m \quad \forall \zeta \in B_{r}
$$

for some positive constant $c_{4}$. The estimates (6.16) clearly apply to the function $v:=\eta u$. By using the properties (6.17), we have

$$
\|L v\|_{L^{p}\left(B_{r}\right)} \leq\|\eta L u\|_{L^{p}\left(B_{r}\right)}+\frac{c_{5}}{\rho^{2} r^{2}}\|u\|_{L^{p}\left(B_{\rho^{\prime} r}\right)}+\frac{c_{5}}{\rho r} \sum_{i=1}^{m}\left\|u_{x_{i}}\right\|_{L^{p}\left(B_{\rho^{\prime} r}\right)}
$$

so that, from (6.16) it follows that

$$
\begin{aligned}
\sum_{i, j=1}^{m}\left\|u_{x_{i} x_{j}}\right\|_{L^{p}\left(B_{\rho r}\right)}+ & \|Y u\|_{L^{p}\left(B_{\rho r}\right)} \leq \\
& c_{6}\left(\|L u\|_{L^{p}\left(B_{r}\right)}+\frac{1}{\rho^{2} r^{2}}\|u\|_{L^{p}\left(B_{\rho^{\prime} r}\right)}+\frac{1}{\rho r} \sum_{i=1}^{m}\left\|u_{x_{i}}\right\|_{L^{p}\left(B_{\rho^{\prime} r}\right)}\right) .
\end{aligned}
$$

In order to conclude the proof, we have to remove the terms $\left\|u_{x_{i}}\right\|_{L^{p}\left(B_{\rho^{\prime} r}\right)}$ from the right hand side of the above inequality. To this aim, we use a standard interpolation formula:

$$
\rho r\left\|u_{x_{h}}\right\|_{L^{p}\left(B_{\rho r}\right)} \leq \varepsilon(\rho r)^{2}\left\|u_{x_{h} x_{h}}\right\|_{L^{p}\left(B_{\rho r}\right)}+\frac{c_{p}}{\varepsilon}\|u\|_{L^{p}\left(B_{\rho r}\right)},
$$


(see, for instance, Theorem 7.27 in [16]). By using the above inequality in (6.18) we get

$$
\begin{aligned}
& \sup _{0<\rho<1}(\rho r)^{2} \sum_{i, j=1}^{m}\left\|u_{x_{i} x_{j}}\right\|_{L^{p}\left(B_{\rho r}\right)}+\sup _{0<\rho<1}(\rho r)^{2}\|Y u\|_{L^{p}\left(B_{\rho r}\right)} \leq \\
& \quad c_{7}\left(r^{2}\|L u\|_{L^{p}\left(B_{r}\right)}+\sup _{0<\rho<1}\|u\|_{L^{p}\left(B_{\rho r}\right)}+\sup _{0<\rho<1}(\rho r) \sum_{h=1}^{m}\left\|u_{h}\right\|_{L^{p}\left(B_{\rho r}\right)}\right) \leq \\
& \quad c_{7}\left(r^{2}\|L u\|_{L^{p}\left(B_{r}\right)}+\left(1+\frac{c_{p}}{\varepsilon}\right) \sup _{0<\rho<1}\|u\|_{L^{p}\left(B_{\rho r}\right)}+\varepsilon \sup _{0<\rho<1}(\rho r)^{2} \sum_{h, k=1}^{m}\left\|u_{x_{h} x_{k}}\right\|_{L^{p}\left(B_{\rho r}\right)}\right) .
\end{aligned}
$$

Hence, if $\varepsilon$ is sufficiently small, we have

$$
\sup _{0<\rho<1}(\rho r)^{2} \sum_{i, j=1}^{m}\left\|u_{x_{i} x_{j}}\right\|_{L^{p}\left(B_{\rho r}\right)}+\sup _{0<\rho<1}(\rho r)^{2}\|Y u\|_{L^{p}\left(B_{\rho r}\right)} \leq c_{8}\left(\|L u\|_{L^{p}\left(B_{r}\right)}+\|u\|_{L^{p}\left(B_{r}\right)}\right)
$$

The estimate (6.15) then follows by taking $\rho=\frac{1}{2}$ in the above inequality.

Proof of Theorem 2.1. We first prove

$$
\|u\|_{C_{B}^{1, \alpha}(\Omega)} \leq C\|u\|_{\mathcal{S}^{p}(\Omega)}, \quad \text { for every } u \in C_{0}^{\infty}(\Omega) .
$$

A standard density argument and the use of a cut-off function plainly gives (2.3).

We denote by $\Gamma$ the fundamental solution of the Kolmogorov operator in (1.2) and we use the standard representation formulas

$$
u(z)=-\int_{\mathbb{R}^{N+1}} \Gamma(z, w) K u(w) d w, \quad \partial_{x_{j}} u(z)=-\int_{\mathbb{R}^{N+1}} \partial_{x_{j}} \Gamma(z, w) K u(w) d w,
$$

for $j=1, \ldots, m$. Aiming to prove (6.19) we recall the following pointwise estimates, that have been proved in [10] (see Proposition 2.7). There exists a positive constant $C_{\Omega}$ such that,

$$
\begin{array}{r}
\Gamma(\zeta, w) \leq \frac{C_{\Omega}}{\left\|w^{-1} \circ \zeta\right\|^{Q}}, \quad|\Gamma(\zeta, w)-\Gamma(\bar{\zeta}, w)| \leq C_{\Omega} \frac{\left\|\zeta^{-1} \circ \bar{\zeta}\right\|}{\left\|w^{-1} \circ \zeta\right\|^{Q+1}}, \\
\left|\partial_{x_{j}} \Gamma(\zeta, w)\right| \leq \frac{C_{\Omega}}{\left\|w^{-1} \circ \zeta\right\|^{Q+1}}, \quad\left|\partial_{x_{j}} \Gamma(\zeta, w)-\partial_{x_{j}} \Gamma(\bar{\zeta}, w)\right| \leq C_{\Omega} \frac{\left\|\zeta^{-1} \circ \bar{\zeta}\right\|}{\left\|w^{-1} \circ \zeta\right\|^{Q+2}},
\end{array}
$$

for every $\zeta, \bar{\zeta}, w \in \Omega$ such that $\left\|\zeta^{-1} \circ \bar{\zeta}\right\| \leq M\left\|w^{-1} \circ \zeta\right\|$ and $j=1, \ldots m$. The estimate (6.19) then follows from the same argument used in the proof of Theorem 2.1 in [33] (we refer to [33] for the details). 


\section{References}

[1] G. BARLES, Convergence of numerical schemes for degenerate parabolic equations arising in finance theory, in Numerical methods in finance, Cambridge Univ. Press, Cambridge, 1997, pp. 1-21.

[2] J. Barraquand And T. Pudet, Pricing of American path-dependent contingent claims, Math. Finance, 6 (1996), pp. 17-51.

[3] E. Barucci, S. Polidoro, and V. Vespri, Some results on partial differential equations and Asian options, Math. Models Methods Appl. Sci., 11 (2001), pp. 475497.

[4] A. Bensoussan, On the theory of option pricing, Acta Appl. Math., 2 (1984), pp. 139-158.

[5] A. Bensoussan And J.-L. Lions, Applications of variational inequalities in stochastic control, vol. 12 of Studies in Mathematics and its Applications, NorthHolland Publishing Co., Amsterdam, 1982. Translated from the French.

[6] M. Bramanti, M. C. Cerutti, And M. Manfredini, L ${ }^{p}$ estimates for some ultraparabolic operators with discontinuous coefficients, J. Math. Anal. Appl., 200 (1996), pp. 332-354.

[7] M. G. Crandall, H. Ishit, And P.-L. Lions, User's guide to viscosity solutions of second order partial differential equations, Bull. Amer. Math. Soc. (N.S.), 27 (1992), pp. $1-67$.

[8] M. Dai AND Y. K. KWoK, Characterization of optimal stopping regions of American Asian and lookback options, Mathematical Finance, 16 (2006).

[9] M. Di Francesco And A. Pascucci, On the complete model with stochastic volatility by Hobson and Rogers, Proc. R. Soc. Lond. Ser. A Math. Phys. Eng. Sci., 460 (2004), pp. 3327-3338.

[10] M. Di Francesco And S. Polidoro, Schauder estimates, Harnack inequality and Gaussian lower bound for Kolmogorov type operators in non-divergence form, Advances in Differential Equations, (2006), pp. 1261-1320.

[11] G. B. Folland, Subelliptic estimates and function spaces on nilpotent Lie groups, Ark. Mat., 13 (1975), pp. 161-207.

[12] P. Foschi And A. Pascucci, Path dependent volatility, preprint AMS Acta, (2007).

[13] A. Friedman, Parabolic variational inequalities in one space dimension and smoothness of the free boundary, J. Functional Analysis, 18 (1975), pp. 151-176. 
[14] _ Variational principles and free-boundary problems, Pure and Applied Mathematics, John Wiley \& Sons Inc., New York, 1982. A Wiley-Interscience Publication.

[15] —_, Variational principles and free-boundary problems, Robert E. Krieger Publishing Co. Inc., Malabar, FL, second ed., 1988.

[16] D. Gilbarg And N. S. Trudinger, Elliptic partial differential equations of second order, Springer-Verlag, Berlin, 1977. Grundlehren der Mathematischen Wissenschaften, Vol. 224.

[17] A. T. Hansen And P. L. Jorgensen, Analytical valuation of American-style Asian options, , Manag. Sci., 46(8) (2000), p. 11161136.

[18] D. G. Hobson And L. C. G. Rogers, Complete models with stochastic volatility, Math. Finance, 8 (1998), pp. 27-48.

[19] L. Hörmander, Hypoelliptic second order differential equations, Acta Math., 119 (1967), pp. 147-171.

[20] P. Jaillet, D. Lamberton, and B. Lapeyre, Variational inequalities and the pricing of American options, Acta Appl. Math., 21 (1990), pp. 263-289.

[21] L. JiAng AND M. DAI, Convergence of binomial tree methods for European/American path-dependent options., SIAM J. Numer. Anal., 42 (2004), pp. 1094 1109.

[22] I. Karatzas, On the pricing of American options, Appl. Math. Optim., 17 (1988), pp. 37-60.

[23] D. Kinderlehrer and G. Stampacchia, An introduction to variational inequalities and their applications, vol. 31 of Classics in Applied Mathematics, Society for Industrial and Applied Mathematics (SIAM), Philadelphia, PA, 2000. Reprint of the 1980 original.

[24] E. Lanconelli And A. Pascucci, On the fundamental solution for hypoelliptic second order partial differential equations with non-negative characteristic form, Ricerche Mat., 48 (1999), pp. 81-106.

[25] E. Lanconelli And S. Polidoro, On a class of hypoelliptic evolution operators, Rend. Sem. Mat. Univ. Politec. Torino, 52 (1994), pp. 29-63.

[26] M. Manfredini, The Dirichlet problem for a class of ultraparabolic equations, Adv. Differential Equations, 2 (1997), pp. 831-866.

[27] M. Manfredini And S. Polidoro, Interior regularity for weak solutions of ultraparabolic equations in divergence form with discontinuous coefficients, Boll. Unione Mat. Ital. Sez. B Artic. Ric. Mat. (8), 1 (1998), pp. 651-675. 
[28] M. D. MARCozzI, On the valuation of Asian options by variational methods., SIAM J. Sci. Comput., 24 (2003), pp. 1124-1140.

[29] G. H. MeYer, On pricing American and Asian options with PDE methods, Acta Math. Univ. Comenian. (N.S.), 70 (2000), pp. 153-165.

[30] A. Nagel, E. M. Stein, And S. Wainger, Balls and metrics defined by vector fields. I. Basic properties, Acta Math., 155 (1985), pp. 103-147.

[31] A. PASCUCCI, Free boundary and optimal stopping problems for American Asian options, preprint AMS Acta, (2007).

[32] G. Peskir And A. Shiryaev, Optimal stopping and free-boundary problems., Lectures in Mathematics, ETH Zürich. Basel: Birkhäuser. xxii, 500 p. EUR 48.00/net; SFR $78.00,2006$.

[33] S. Polidoro And M. A. RagusA, Sobolev-Morrey spaces related to an ultraparabolic equation, Manuscripta Math., 96 (1998), pp. 371-392.

[34] L. Rogers And Z. Shi, The value of an Asian option., J. Appl. Probab., 32 (1995), pp. 1077-1088.

[35] L. P. Rothschild And E. M. Stein, Hypoelliptic differential operators and nilpotent groups, Acta Math., 137 (1976), pp. 247-320. 